\title{
Predicting outcomes in patients deferred for gastrostomy insertion using either the Sheffield gastrostomy score or the Levine score
}

\author{
M. Kurien, J. S. Leeds, H. E. Robson, J. Grant, M. E. McAlindon and D. Sanders \\ Department of Gastroenterology, Royal Hallamshire Hospital, Sheffield, UK
}

Gastrostomy insertion is widely accepted as the best means of providing medium and long term enteral nutrition. There is limited data on outcomes in patients referred for gastrostomy insertion that are either refused or do not survive until PEG insertion. Scoring systems such as the Levine score have been created to try and predict survival rates over 1 year for patients admitted acutely. The aim of this study was to determine the clinical characteristics and outcomes of patients in whom gastrostomy insertion was not felt to be appropriate on clinical grounds and the Levine score and the Sheffield gastrostomy score (SGS) applied.

All patients referred for gastrostomy insertion in our institute are reviewed by the gastrostomy nurse specialist and prospectively included in a database. Demographics, referral indication, biochemical profile and outcome data are also included in the database. Gastrostomy referrals from October 2003 to September 2010 were analysed and actual mortality rate was compared to the expected mortality rate predicted by the SGS and Levine score.

304 patients were included in the study (median age 77 years, 175 males) all of whom were referred for consideration of gastrostomy insertion but were ultimately not inserted. The 3 top reasons for non-placement were patient too ill (29.3\%), oral intake restarted (18.1\%) and patient died (13.5\%). Overall mortality at 30 days and 1 year was 122/304 (40.1\%) and 226/304 (74.3\%) respectively. The highest 30 day mortality was seen in patients with cognitive impairment $12 / 17(70.6 \%)$ and followed by dysphagic stroke 53/105 $(50.5 \%)$. The main predictors of 30 day mortality were age $>60$ (OR 7.2, 3.3-15.8, $p<0.001$ ) and albumin $<25$ (OR 2.5, 1.5-5.3, $p=0.01$ ). 1 year mortality as predicted by Levine score or Sheffield score is shown in the table.

The Sheffield gastrostomy score is more accurate at predicting 1-year mortality in patients deferred for gastrostomy insertion than the Levine score. This may suggest that the Sheffield PEG score could be used in the pre-assessment of patients who are being referred for PEG as a means of estimating/predicting mortality for those involved in the decision making process.

\begin{tabular}{lrrc}
\hline & Expected mortality & Actual Mortality & $\mathrm{P}$ \\
\hline Sheffield 0 & $15 / 39(38.5 \%)$ & $5 / 9(55.6 \%)$ & 0.35 \\
Sheffield 1 & $53 / 100(53.0 \%)$ & $33 / 51(64.7 \%)$ & 0.17 \\
Sheffield 2 & $68 / 94(72.3 \%)$ & $67 / 91(73.6 \%)$ & 0.84 \\
Sheffield 3 & $40 / 51(78.4 \%)$ & $82 / 93(88.2 \%)$ & 0.12 \\
Levine 0 - 1 & $110 / 799(13.8 \%)$ & $64 / 105(33.9 \%)$ & $<0.001$ \\
Levine 2 & $130 / 719(18.1 \%)$ & $35 / 43(81.4 \%)$ & $<0.001$ \\
Levine 3 & $180 / 563(31.9 \%)$ & $98 / 112(87.5 \%)$ & $<0.001$ \\
Levine 4+ & $299 / 647(46.2 \%)$ & $5 / 5(100 \%)$ & 0.06 \\
\hline
\end{tabular}

